\title{
Osseous Heart- A Rare Case of Metastatic Osteosarcoma to the Heart
}

\begin{abstract}
Arnaldo A Nieves Ortiz ${ }^{1 *}$, Kyomara Hernández Moya ${ }^{1}$, Arnaldo Rojas Figueroa ${ }^{1}$, Vanessa Fonseca Ferrer ${ }^{1}$, Hernan Gonzalez Monroig ${ }^{2}$, Jonathan Martinez Feshold ${ }^{1}$, William Marrero ${ }^{1}$, Gabriela Torres Torres ${ }^{1}$, Alexis Cruz Chacón ${ }^{2}$, Rafael Rivera Berrios ${ }^{3}$ and Teodosio Rucabado ${ }^{4}$
\end{abstract}

${ }^{1}$ Internal Medicine Department, San Juan City Hospital, Puerto Rico

${ }^{2}$ Hematology/Oncology Fellowship, San Juan City Hospital/VA Caribbean Healthcare System, Puerto Rico

${ }^{3}$ Cardiovascular Department Fellowship, Saint Luke's Episcopal Medical Center, Puerto Rico

${ }^{4}$ Cardiovascular Department, San Juan City Hospital, Puerto Rico

*Corresponding author: Arnaldo A Nieves Ortiz, Internal Medicine Department,

San Juan City Hospital, San Juan, Puerto Rico.

Received Date: July 21, 2021

Published Date: August 05, 2021

\begin{abstract}
Osteosarcomas are uncommon malignant tumors of the bone that arise from the production of osteoid or immature bone by the malignant cells. In rare occasions this tumor metastasizes to other organs including the brain and lungs. Although hematogenous spread of osteosarcoma is well known, cardiovascular metastasis is seldomly reported. Most cases of metastatic osteosarcoma to the heart involve the myocardium rather than the pericardium. The right side of the heart is affected in $20-30 \%$ of cases and the left side in $10-33 \%$ of reported cases. Only $5 \%$ of cases affect the endocardium or chamber cavities. Clinical signs and symptoms of metastatic cardiac neoplasms include dyspnea, cough, chest pain, palpitations, superior vena cava syndrome, right ventricular outflow tract obstruction and pericardial effusion [1].
\end{abstract}

Keywords: Cardiac Osteosarcomas; GM-CSF autoantibodies; Autoimmune

\section{Case Presentation}

We present the case of a $48 \mathrm{y} / \mathrm{o}$ male with history of osteosarcoma of the left femur s/p 5 cycles of neoadjuvant chemotherapy with Doxorubicin and Cisplatin on May 2020, who presented with complains of left femur pain. Upon review of system, patient denied chest pain, SOB, dyspnea on exertion, palpitations, or syncope. Physical exam remarkable for left knee decreased range of motion and strength $4 / 5$. Laboratories showed normocytic normochromic anemia and mild thrombocytopenia. Left leg X-ray showed a $9.9 \mathrm{~cm}$ sclerotic lesion with associated cortical thickening and prominent sunburst reaction at the femoral shaft to the metaphysis worrisome for aggressive osteosarcoma. Knee CT scan without IV contrast shows sunburst periosteal reaction measuring $13.9 \mathrm{~cm}$ long x $6.1 \mathrm{~cm}$ transverse x $5.8 \mathrm{~cm}$. On December 2020, Chest CT shows enlarged pulmonary nodules consistent with metastasis. Patient was referred to osteosarcoma specialist who recommended Etoposide, Ifosfamide, Mesna and high dose methotrexate. Prior to initiation of induction chemotherapy, transthoracic echocardiography was done and showed a $11.25 \mathrm{~cm}$ mass filling the right ventricle with preserved EF. Cardiac MRI results showed homogenously enhancing intracavitary mass centered at the apex of the right ventricle measuring approximately $3.2 \times 4.2 \mathrm{~cm} \times 5.0$ $\mathrm{cm}$, an internal and eccentric non-enhancing hypointense signal 
representative of an ossifying focus characteristic of osteosarcoma metastasis. Bone marrow biopsy showed no evidence of metastatic osteosarcoma. Patient was initiated on 2 cycles of chemotherapy, with improved clinical response (Figures 1-3).
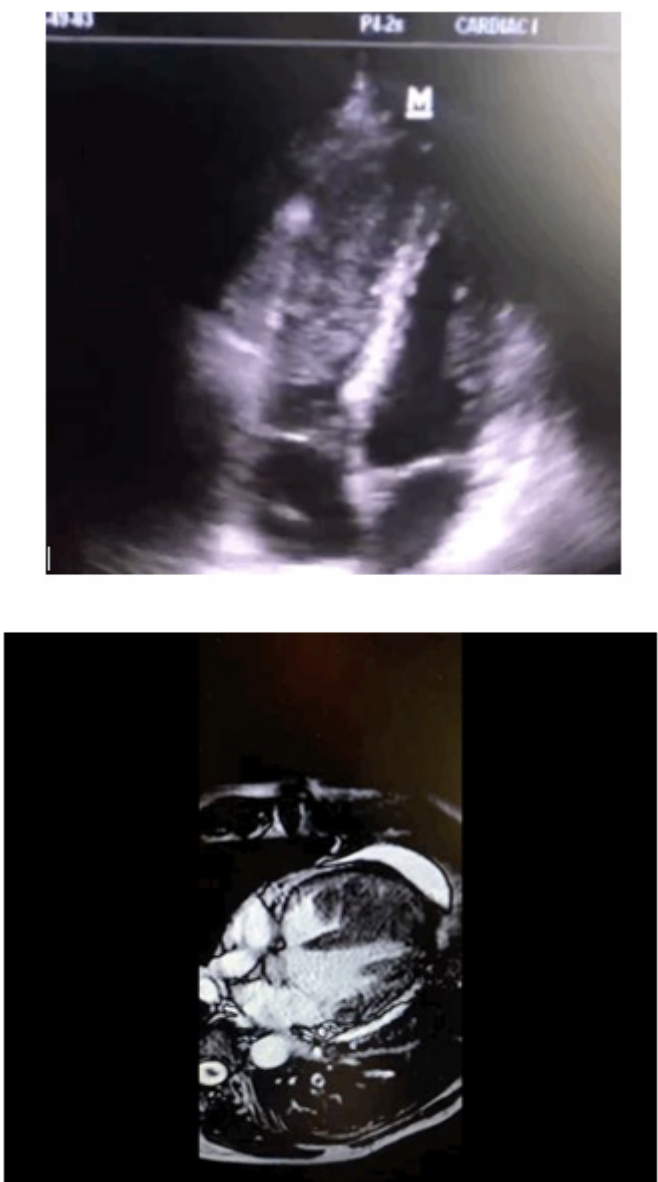

Figure 1A: Left Femur Biopsy: Findings confirmed high grade osteosarcoma.

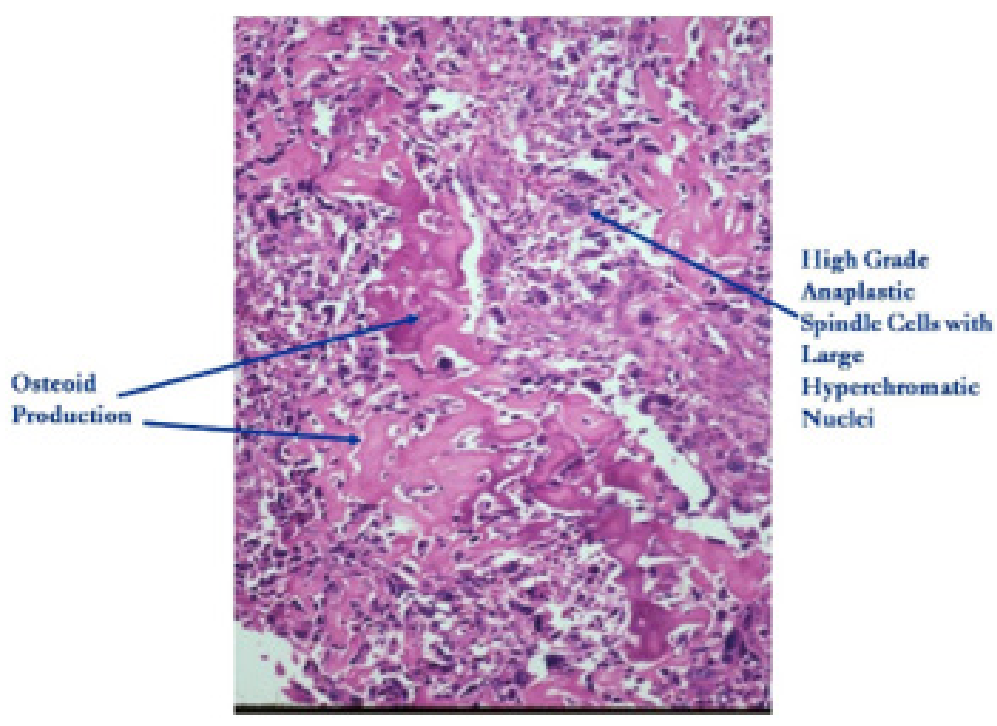

Figure 1B: Histological findings consistent with Osteosarcoma.

Figure 1: Collection canister from WLL fluid with progressive translucency of fluid from 1 through 4. 


\section{Conclusion}

Osteosarcomas are malignant tumors that most frequently involves the long bones of young adults within the second and third decades of life. The clinical characteristics involve hemodynamic compromise or precordial abnormality, with magnetic resonance imaging emerging as the gold standard for diagnosis. Osteosarcoma metastasis most commonly occur in the lungs. Osteosarcoma very rarely metastasizes to the heart's right ventricle, all of them in the RV outflow tract. Although surgery is generally contraindicated in the presence of metastatic disease, the role of surgical palliative of intracardiac tumor to relieve obstruction and to prevent embolization is considered. However, even with successful surgical resection with or without chemotherapy, the prognosis of patients with osteosarcoma is very poor [2]. Despite being extremely rare to find antemortem involvement in the heart, $20 \%$ of autopsies show involvement in the heart. In this case, the enhanced resolution of echocardiogram and Chest CT scan lead to detection of cardiovascular involvement.

\section{Acknowledgment}

We would like to acknowledge the department of Internal Medicine Program, San Juan City Hospital. We have no conflict of interest to declare. This statement is to certify that all authors have seen and approved the manuscript being submitted.

\section{Conflict of Interest}

No Conflict of Interest.

\section{References}

1. Maleki M, Mozaffari K, Givtaj N, Tatina A, Bahadorian B (2013) Metastatic osteosarcoma and heart: a rare involvement in an unusual cardiac location. Heart Asia 5(1): 120-121.

2. Abdelfatah Elasfar, Ahmed Khalifa, Abdulaziz AlGhamdi, Rizwan Khalid, Mohamed Ibrahim, et al. (2013) Asymptomatic metastatic osteosarcoma to the right ventricle: Case report and review of the literature. J Saudi Heart Assoc 25(1): 39-42. 